\title{
ADSORÇÃO DE POLIHEXAMETILENO BIGUANIDA (PHMB) EM MEMBRANAS POROSAS DE QUITOSANA E ALGINATO
}

\author{
CECILIA. Z. BUENO ${ }^{1}$, PAULO T. V. ROSA ${ }^{2}$ E ÂNGELA. M. MORAES ${ }^{1}$ \\ ${ }^{1}$ Universidade Estadual de Campinas, Faculdade de Engenharia Química, Departamento de \\ Engenharia de Materiais e Bioprocessos \\ ${ }^{2}$ Universidade Estadual de Campinas, Instituto de Química \\ E-mail para contato: ammoraes@feq.unicamp.br
}

\begin{abstract}
RESUMO - O PHMB é um dos agentes antimicrobianos mais promissores da atualidade devido à sua eficácia contra um amplo espectro de bactérias, podendo ser impregnado a vários produtos, como curativos. A adsorção por via úmida é um dos métodos de impregnação mais comuns. Neste trabalho, estudou-se a adsorção de PHMB em membranas porosas de quitosana-alginato destinadas ao tratamento de lesões de pele. Empregou-se uma ampla faixa de concentrações de soluções aquosas de PHMB (de $4,0 \times 10^{-7}$ a $7,6 \times 10^{-2} \mathrm{~mol} / \mathrm{L}$ ), para as quais foi determinada a isoterma de adsorção a $37^{\circ} \mathrm{C}$. Os dados obtidos foram ajustados pelos modelos de Freundlich e Zhu e Gu (1989). Observou-se que a capacidade de adsorção aumentou ao longo de todo o experimento, possibilitando impregnar grandes quantidades de PHMB (máximo experimental obtido de cerca de $6 \mathrm{~g} / \mathrm{g}$ nas condições testadas). Foi observado um comportamento típico de isoterma de Langmuir a baixas concentrações, a partir do qual o sistema seguiu um comportamento de múltiplos platôs, característico da adsorção de alguns surfatantes em interfaces sólido/líquido.
\end{abstract}

\section{INTRODUÇÃO}

O PHMB (polihexametileno biguanida) é um polímero que pode se apresentar na forma catiônica abaixo de pH igual a 10,96 (O’Malley et al., 2006). Este composto tem excelente atividade antimicrobiana e pode ser incorporado a uma variedade de produtos, incluindo curativos, soluções de limpeza de lentes de contato, produtos para assepsia cirúrgica, tecidos, sabonetes, cosméticos e produtos para limpeza de piscinas (Gao e Cranston, 2008; Arch Biocides, 2008; De Paula et al., 2011). A variedade de possibilidades de aplicações do PHMB se deve ao fato de ele ser solúvel em água, estável ao calor, inodoro, compatível com ampla faixa de valores de $\mathrm{pH}$ (entre 1,0 e 9,0), além de apresentar baixa toxicidade, custo razoável e ser de baixo impacto ambiental (De Paula et al., 2011). A estrutura química do PHMB é mostrada na Figura 1.

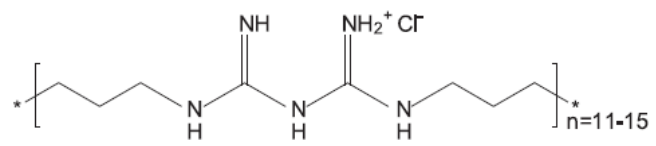

Figura 1 - Fórmula estrutural do PHMB (Gao e Cranston, 2008). 


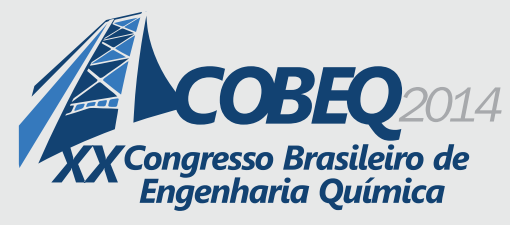

19 a 22 de outubro de 2014
Florianópolis/SC

Neste trabalho, estudou-se a adsorção de PHMB em membranas porosas de quitosana-alginato (Q-A), objetivando-se o uso do material obtido para o tratamento de lesões de pele. A quitosana e o alginato são polissacarídeos biocompatíveis e biodegradáveis de baixo custo que podem formar complexos iônicos estáveis em determinadas condições de $\mathrm{pH}$. Tais complexos podem ser moldados na forma de membranas densas. A adição de um surfatante biocompatível, como o Pluronic F68, durante o preparo da mistura polimérica sob agitação provoca o surgimento de bolhas de ar, que ficam retidas na estrutura formada. Após a secagem do material, é possível obter membranas porosas com boa capacidade de absorção de fluidos e características adequadas para a cobertura direta de lesões de pele (Bueno e Moraes, 2011). A aplicabilidade de tais dispositivos poderia ser aumentada pela adição de PHMB, tornando os curativos particularmente atrativos no caso de necessidade de controle de microrganismos presentes em feridas infectadas.

Um dos métodos mais comuns de incorporação de agentes ativos a dispositivos biomédicos é a incorporação a úmido, que consiste em mergulhar o dispositivo pronto em uma solução do agente ativo, o qual é adsorvido pelo material. Idealmente, se a matriz do dispositivo interagir com o agente bioativo, pode-se ter maior controle sobre sua cinética de liberação após o intumescimento em meios fisiológicos do que nos casos em que se verifica a simples retenção física.

O PHMB apresenta comportamento típico de surfatante devido à sua estrutura anfifílica. A estrutura alternada de segmentos hidrofílicos e hidrofóbicos faz com que as moléculas de PHMB se arranjem de maneira específica em solução aquosa, na qual os segmentos hidrofóbicos de hexametileno se direcionam para o centro de uma esfera, e os segmentos hidrofílicos protonados de biguanida se direcionam para fora de uma esfera. O resultado é uma estrutura similar a uma micela típica, mas formada por segmentos de uma cadeia polimérica, e não por moléculas independentes adjacentes (De Paula et al., 2011). Assim, este fato deve ser levado em consideração no estudo de adsorção do PHMB.

Em geral, a adsorção de um surfatante a um sólido pode ser governada por forças eletrostáticas, covalentes, ligações de hidrogênio e interações hidrofóbicas, dependendo do tipo de sistema em questão. A energia total de interação na adsorção é normalmente o resultado cumulativo de algumas ou de todas estas forças. A isoterma de adsorção de um surfatante pode ser classificada basicamente em três tipos, de acordo com o seu formato: tipo Langmuir (L), tipo S (S), ou tipo dois platôs (L-S), com características de ambas as isotermas anteriores (Zhang e Somasundaran, 2006). A equação proposta por Zhu e Gu (1989) é um modelo geral para a adsorção de surfatantes em interfaces sólido-líquido e engloba esses três tipos de isotermas. Este modelo é mostrado na Equação 1.

$$
q=\frac{q_{\max } k_{1} C\left(\frac{1}{n}+k_{2} C^{n-1}\right)}{1+k_{1} C\left(1+k_{2} C^{n-1}\right)}
$$

onde: $q$ é a quantidade de surfatante adsorvida por unidade de massa do adsorvente; $q_{\max }$ é a capacidade máxima de adsorção do adsorvente; $C$ é a concentração de surfatante no equilíbrio; $k_{1}$ é a constante de equilíbrio da primeira etapa da adsorção, quando há a interação direta entre o surfatante e a superfície adsorvente; $k_{2}$ é a constante de equilíbrio da segunda etapa da adsorção, na qual ocorre a 


\section{9 a 22 de outubro de 2014 \\ Florianópolis/SC}

formação de hemimicelas (agregados de moléculas que se estruturam formando uma monocamada sobre a superfície sólida em decorrência da interação entre as moléculas de surfatante adsorvidas e as moléculas de surfatante em solução) a partir da concentração hemimicelar crítica (CHC), etapa esta na qual ocorre um aumento significativo da quantidade de moléculas adsorvidas; e $n$ é um parâmetro que se refere ao número de agregação nas hemimicelas, ou seja, o número de moléculas por hemimicela.

Os parâmetros da Equação 1 podem assumir diversos valores. Um dos casos que simplificam esta equação é quando $k_{2}$ tende a zero e $n$ tende a um, o que faz com que a mesma se reduza a uma isoterma tipo Langmuir, como mostrado na Equação 2.

$$
q=\frac{q_{\max } k_{1} C}{1+k_{1} C}
$$

A adsorção de surfatantes em interfaces sólido-líquido, em particular do PHMB em celulose (Blackburn et al., 2006), pode ser também descrita pelo modelo de Freundlich, que prevê a formação de múltiplas camadas em decorrência de interações entre as moléculas adsorvidas. Este modelo descreve bem os sistemas de adsorção altamente heterogêneos, cuja superfície adsorvente possui distribuição irregular de energia, o que possivelmente seria o caso de matrizes de quitosana complexada com alginato. $\mathrm{Na}$ isoterma de Freundlich, mostrada na Equação 3, $k$ é o coeficiente de adsorção de Freundlich e $m$ indica qualitativamente a reatividade dos sítios de ligação do adsorvente.

$$
q=k C^{1 / m}
$$

Neste trabalho, as equações de Zhu e Gu (1989) e Freundlich foram empregadas para modelar o sistema de adsorção de PHMB em membranas porosas de Q-A destinadas ao tratamento de lesões de pele. O objetivo do trabalho foi compreender o mecanismo de adsorção do agente antimicrobiano em solução aquosa na matriz polissacarídica, o qual é um dos métodos de impregnação mais comuns e pode aumentar a eficácia das membranas no tratamento de feridas contaminadas.

\section{MATERIAIS E MÉTODOS}

\subsection{Preparo das membranas}

Para o preparo das membranas de Q-A, utilizou-se quitosana obtida de camarão, com grau de desacetilação de $95 \pm 1 \%$ determinado por titulação (lote $n^{\circ} 068 \mathrm{~K} 00851$ ) e alginato de sódio de baixa viscosidade (lote $\mathrm{n}^{\circ}$ 106K0113), ambos da Sigma Chemical Co. Utilizou-se também Pluronic F68 da Sigma Chemical Co., cloreto de cálcio dihidratado e hidróxido de sódio da Merck KGaA e ácido acético glacial da Synth ${ }^{\circledR}$.

Para a obtenção das membranas, $90 \mathrm{~mL}$ de solução de quitosana a $1 \%(\mathrm{~m} / \mathrm{v})$ em ácido acético a $2 \%(\mathrm{~m} / \mathrm{v})$ foram adicionados a $180 \mathrm{~mL}$ de solução de alginato a 0,5\% (m/v) contendo Pluronic F68 a $0,02 \%(\mathrm{~m} / \mathrm{v})$. A adição foi feita com auxílio de uma bomba peristáltica (Minipuls 3, Gilson), a uma vazão de $200 \mathrm{~mL} / \mathrm{h}$. A reação entre os polímeros ocorreu em um reator de aço inoxidável encamisado 


\section{9 a 22 de outubro de 2014 \\ Florianópolis/SC}

sob agitação a $500 \mathrm{rpm}$ (agitador mecânico Q-251D, Quimis) e a $25^{\circ} \mathrm{C}$ (banho termostático Q-214M2, Quimis). Ao término da mistura entre as soluções, aumentou-se a taxa de agitação para $1000 \mathrm{rpm}$ e a mistura foi agitada por $10 \mathrm{~min}$. Em seguida, adicionou-se $\mathrm{NaOH}$ a $1 \mathrm{M}$ para elevar o $\mathrm{pH}$ a aproximadamente 5,3, mantendo-se a agitação do sistema por mais $10 \mathrm{~min}$. Por fim, foram adicionados 3,6 mL de solução de $\mathrm{CaCl}_{2}$ a $2 \%$ e o sistema permaneceu sob agitação por mais $10 \mathrm{~min}$. A mistura obtida foi dividida igualmente em termos mássicos em duas placas de Petri de poliestireno de $15 \mathrm{~cm}$ de diâmetro e levada à estufa com circulação de ar $\left(410 \mathrm{D}\right.$, Nova Ética) a $60^{\circ} \mathrm{C}$ até a secagem do material. Em seguida, cada membrana foi imersa por $30 \mathrm{~min}$ em $150 \mathrm{~mL} \mathrm{de} \mathrm{CaCl}_{2}$ a $2 \%$, seguida de mais duas imersões de 30 minutos em $200 \mathrm{~mL}$ de água. A secagem final foi realizada em estufa a $37^{\circ} \mathrm{C}$ por 6 horas, sendo as bordas das membranas presas para evitar encolhimento.

\subsection{Análise da adsorção de PHMB}

Para a determinação da isoterma de adsorção de PHMB nos dispositivos de Q-A, amostras de membranas medindo $4 \mathrm{~cm} \times 1 \mathrm{~cm}$ foram imersas em $15 \mathrm{~mL}$ de soluções aquosas de PHMB de diversas concentrações (variando de $4,0 \times 10^{-7}$ a $7,6 \times 10^{-2} \mathrm{~mol} / \mathrm{L}$ ) a $37^{\circ} \mathrm{C}$ e $100 \mathrm{rpm}$ por 24 horas, sendo este tempo suficiente para se atingir o equilíbrio de adsorção, como verificado em ensaios preliminares. Ao final do ensaio, as concentrações de PHMB na fase aquosa foram determinadas através da medida da absorbância das soluções a $235 \mathrm{~nm}$. As curvas de adsorção obtidas foram ajustadas de acordo com os modelos de Freundlich e Zhu e Gu (1989). Os experimentos foram realizados em duplicata.

\subsection{Determinação da eficiência de incorporação de PHMB nas membranas}

A eficiência de incorporação $(E f)$ do PHMB por adsorção às membranas de Q-A foi calculada segundo a Equação 4, em que $P H M B_{\text {inicial }}$ é a massa total de PHMB disponível em solução no início da impregnação e $P H M B_{\text {incorporado }}$ refere-se à massa de PHMB adsorvido.

$$
E f=\frac{P H M B_{\text {incorporad }}}{P H M B_{\text {inicial }}} \times 100
$$

\section{RESULTADOS E DISCUSSÃO}

A análise do desempenho da matriz no que tange à adsorção do composto bioativo que se deseja incorporar é de grande relevância. As amostras de membranas expostas às soluções de PHMB em diferentes concentrações atingiram as eficiências de incorporação do agente antimicrobiano mostradas na Figura 1. Pode-se observar uma tendência de diminuição exponencial da eficiência de incorporação com o aumento da concentração de PHMB. A eficiência inicial a baixas concentrações, que era de cerca de $80 \%$ passou muito rapidamente a cerca de $7 \%$, quando se atingiram concentrações de PHMB entre 0,008 e $0,012 \mathrm{~mol} / \mathrm{L}$. A partir de $0,012 \mathrm{~mol} / \mathrm{L}$, notou-se que a eficiência de incorporação se manteve entre 5 a 7\%. Esta diminuição é esperada nos casos em que a retenção do composto na matriz se dá por adsorção, ocorrendo de forma mais abrupta a baixas concentrações se a adsorção ocorre em monocamada. 


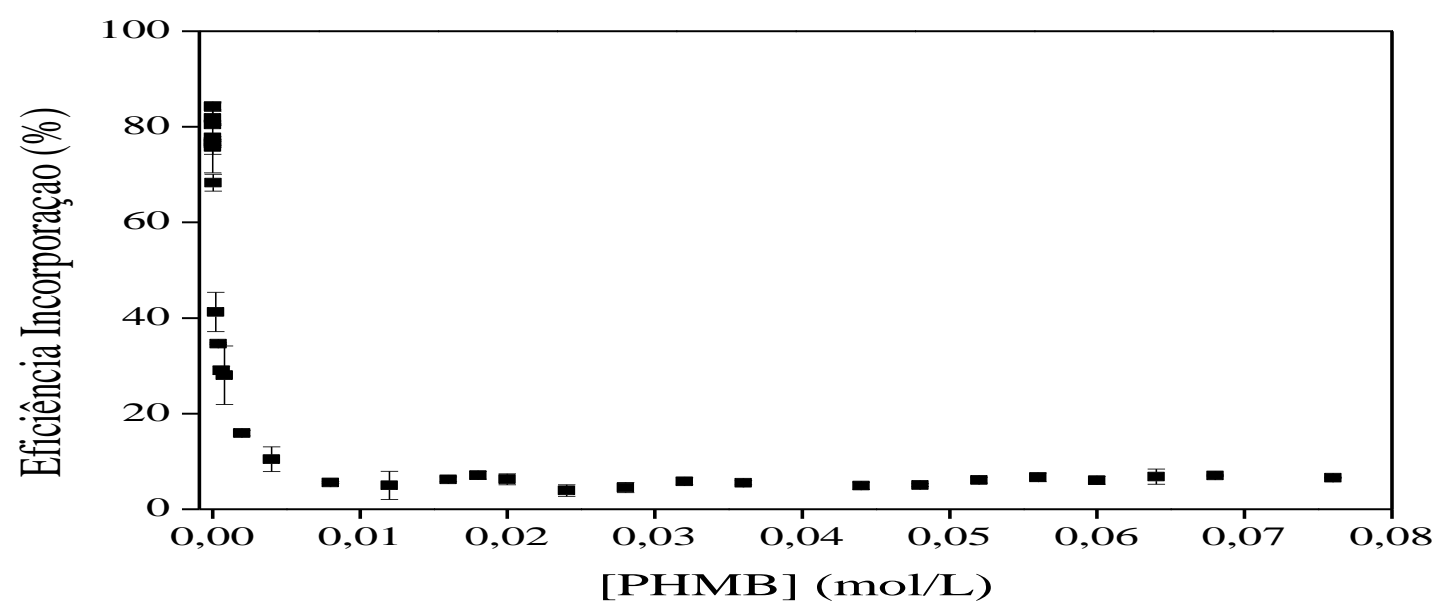

Figura 1 - Eficiência de incorporação em função da concentração inicial de PHMB na fase aquosa.

A forma de adsorção do PHMB nas matrizes de Q-A foi avaliada analisando-se os dados de equilíbrio de adsorção de PHMB e o ajuste dos modelos de Zhu e Gu (1989) e Freundlich a tais resultados em diferentes faixas de concentrações do agente antimicrobiano, conforme mostrado nas Figuras 2 e 3 e sumarizado na Tabela 1. Nestes ensaios, o pH das soluções aquosas variou de 5,7 a 5,9.

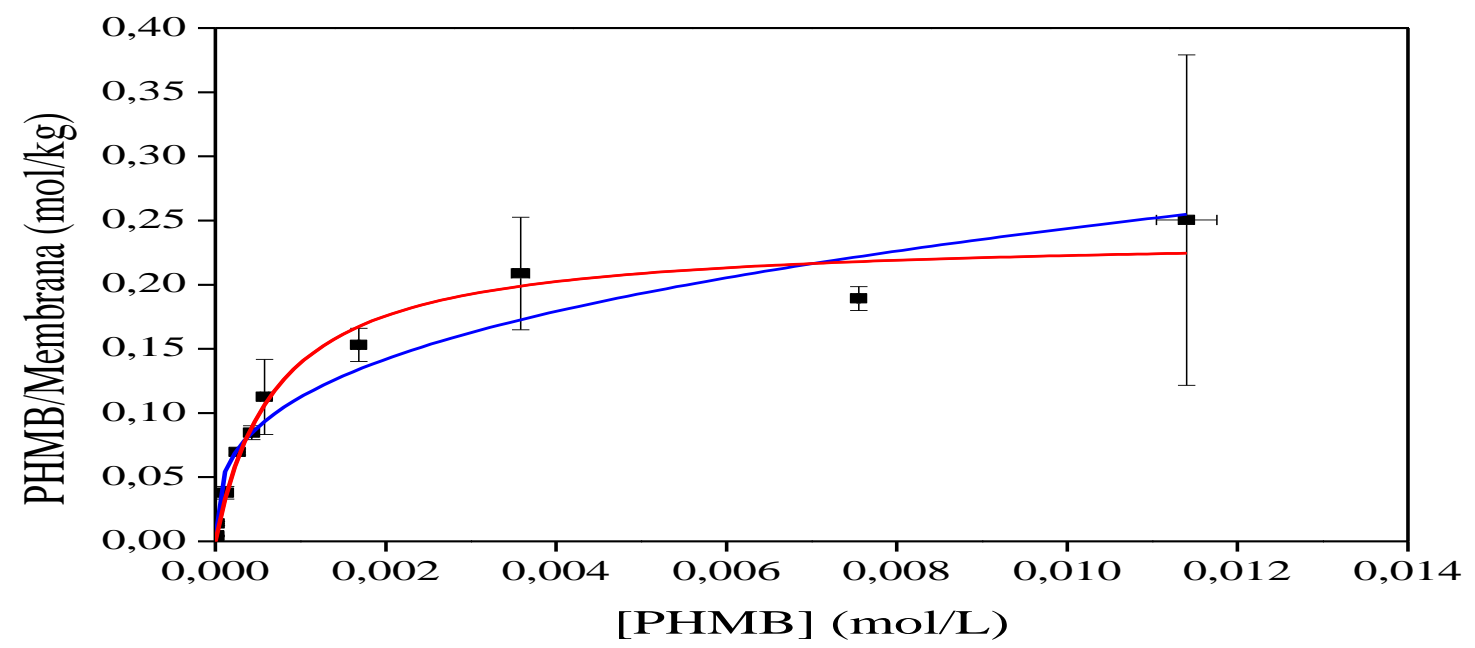

Figura 2 - Ajuste dos modelos de Zhu e Gu (1989) (-) e Freundlich (-) aos dados de adsorção de PHMB em membranas de Q-A a $37^{\circ} \mathrm{C}$ e $100 \mathrm{rpm}$ para concentrações de equilíbrio até $0,012 \mathrm{~mol} / \mathrm{L}$. 


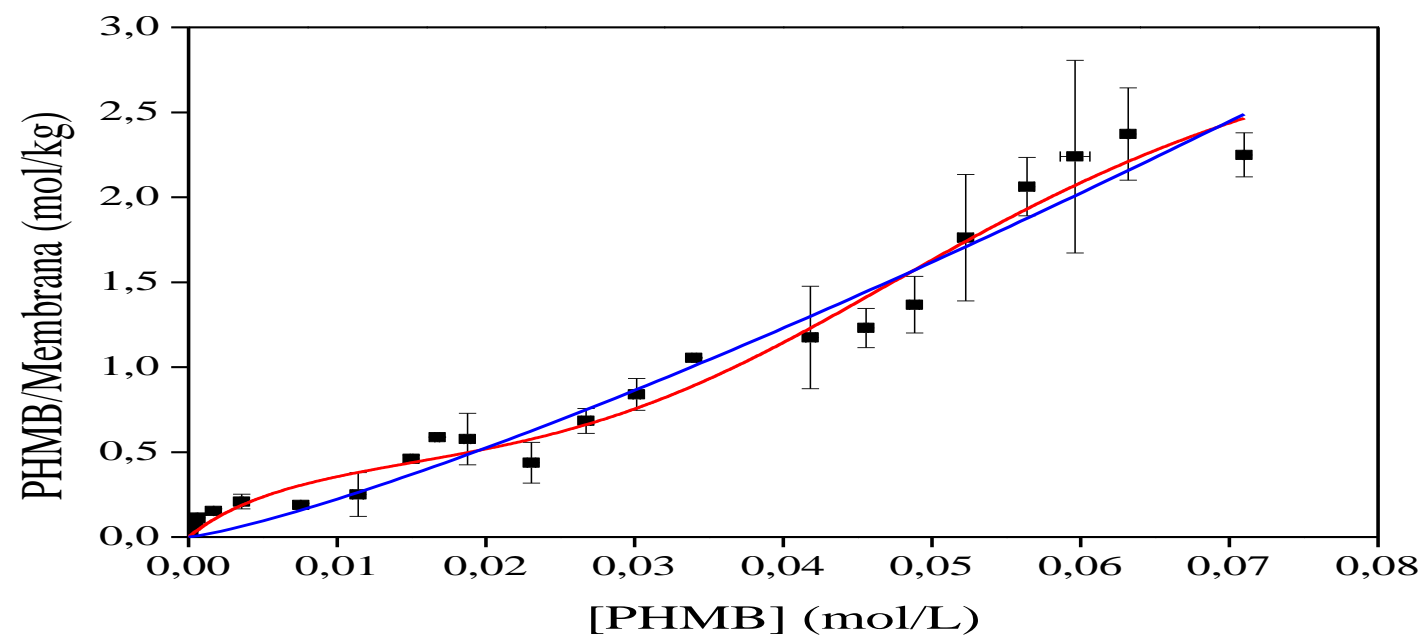

Figura 3 - Ajuste dos modelos de Zhu e Gu (1989) (-) e Freundlich (-) aos dados de adsorção de PHMB em membranas de Q-A a $37^{\circ} \mathrm{C}$ e $100 \mathrm{rpm}$ para concentrações de equilíbrio até $0,076 \mathrm{~mol} / \mathrm{L}$.

Tabela 1 - Parâmetros e coeficientes de determinação obtidos para o ajuste dos diferentes modelos de isotermas aos dados experimentais

\begin{tabular}{|c|c|c|c|c|c|}
\hline \multirow{2}{*}{ Modelo } & \multirow{2}{*}{ Parâmetro } & \multicolumn{2}{|c|}{ [PHMB] até $0,012 \mathrm{~mol} / \mathrm{L}$} & \multicolumn{2}{|c|}{ [PHMB] até $0,076 \mathrm{~mol} / \mathrm{L}$} \\
\hline & & Valor & $\mathrm{R}^{2}$ & Valor & $\mathrm{R}^{2}$ \\
\hline \multirow{2}{*}{ Freundlich } & $k\left(\mathrm{~mol}^{(1-1 / \mathrm{m})} \mathrm{L}^{1 / \mathrm{m}_{\mathrm{kg}}} \mathrm{kg}^{-1}\right)$ & 1,15 & \multirow{2}{*}{0,961} & 64,35 & \multirow{2}{*}{0,975} \\
\hline & $m$ (adimensional) & 2,97 & & 0,81 & \\
\hline \multirow{4}{*}{ Zhu e Gu } & $q_{\max }(\mathrm{mol} / \mathrm{kg})$ & 0,23 & \multirow{4}{*}{0,980} & 3,19 & \multirow{4}{*}{0,983} \\
\hline & $k_{1}(\mathrm{~L} / \mathrm{mol})$ & 1395,00 & & 108,70 & \\
\hline & $k_{2}$ (adimensional) & 0 & & 48753,00 & \\
\hline & $n$ (adimensional) & 0,97 & & 4,68 & \\
\hline
\end{tabular}

A baixas concentrações de PHMB, de até $0,012 \mathrm{~mol} / \mathrm{L}$, o melhor ajuste foi obtido segundo a isoterma de Zhu e $\mathrm{Gu}$ (1989). Nota-se que nesta faixa de concentrações, o formato é típico da isoterma de Langmuir, atingindo-se um platô em aproximadamente $0,23 \mathrm{~mol} / \mathrm{kg}$. Verifica-se também que o parâmetro $k_{2}$ é nulo e $n$ tende a um, tendo-se, assim, o caso limite mostrado na Equação 2. Nesta faixa de concentrações, tem-se este tipo de comportamento provavelmente devido à adsorção do PHMB em monocamada, havendo interações somente entre as moléculas de PHMB adsorvidas e a superfície da membrana. Tais interações seriam provavelmente de ordem eletrostática entre os grupos biguanida do PHMB e as carboxilas do alginato, podendo também ocorrer ligações de hidrogênio entre os grupos biguanida e os grupos - $\mathrm{OH}$ dos polissacarídeos, além de interações hidrofóbicas entre as cadeias de polihexametileno do PHMB e os grupos fracamente hidrofóbicos dos polissacarídeos, como os grupos - $\mathrm{CH}$ da cadeia principal da quitosana e do alginato e os grupos $\mathrm{N}$-acetil da quitosana. 


\section{9 a 22 de outubro de 2014 \\ Florianópolis/SC}

A concentrações maiores de PHMB, de até $0,076 \mathrm{~mol} / \mathrm{L}$, o melhor ajuste foi novamente obtido para a isoterma de Zhu e Gu (1989). O formato da isoterma da Figura 3 é complexo (classificação do tipo L-S, com mais de um platô), devendo-se provavelmente à crescente reorganização das moléculas de PHMB na superfície e à adsorção de moléculas subsequentes, possibilitando atingir a capacidade máxima teórica de adsorção das membranas $\left(q_{\max }\right)$ de cerca de 3,2 mol/kg, equivalente a 8,0 g/g.

Conforme já mencionado, a adsorção de PHMB em celulose foi estudada por Blackburn et al. (2006). Tal matriz adsortiva é comparável às membranas de Q-A, já que a quitosana possui estrutura química semelhante à celulose e o alginato possui grupos carboxila que também podem estar presentes na celulose devido à sua oxidação. Estes autores observaram que, a partir de determinada concentração, as moléculas de PHMB podem interagir entre si através de ligações de hidrogênio entre os grupos biguanida e por interações laterais hidrofóbicas entre as cadeias de polihexametileno, com a formação de hemimicelas (agregação em monocamada) e mesmo de admicelas (agregação do tipo bicamadas ordenadas) na superfície da matriz polissacarídica, havendo, assim, a formação de multicamadas. Os parâmetros da isoterma de Freundlich calculados com base nos dados de adsorção de PHMB em celulose (para concentrações de PHMB até $0,04 \mathrm{~mol} / \mathrm{L}$ ) obtidos por Blackburn et al. (2006) resultam em valores de $k$ e $m$ iguais a, respectivamente, $0,83 \mathrm{~mol}^{(1-1 / \mathrm{m})} \mathrm{L}^{1 / \mathrm{m}_{\mathrm{kg}}} \mathrm{kg}^{-1}$, 76 (com R de 0,980$)$; embora o valor de $m$ seja próximo ao obtido no presente trabalho $(0,81)$ indicando reatividade similar dos sítios de ligação dos adsorventes, o valor do coeficiente de adsorção $k$ é muito superior na matriz de Q-A. Já no modelo de Zhu e Gu (1989), os valores de $q_{\max }, k_{1}, k_{2}$ e $n$ calculados usando os dados de Blackburn et al. (2006) foram de, respectivamente, 20,47 mol/kg, 58,75 L/mol, $1 \mathrm{e}$ 115,08 , com $\mathrm{R}^{2}$ de 0,982 . Em comparação com o presente trabalho, observaram-se as maiores diferenças nos parâmetros $k_{2}$ e $n$, que são referentes à etapa de formação e adsorção na forma de hemimicelas, indicando que estas são maiores no caso da adsorção em celulose.

No sistema Q-A, a concentração hemimicelar crítica $(\mathrm{CHC})$, a partir da qual se formam as hemimicelas, pode ser calculada através da Equação 5 proposta por Zhu et al. (1989).

$$
C H C=\left(\frac{n-2}{n}\right)^{\frac{n}{n-1}} k_{2}^{\frac{-1}{n-1}}
$$

Para o sistema em estudo, obteve-se um valor de $\mathrm{CHC}$ igual a $0,026 \mathrm{~mol} / \mathrm{L}$, o qual pode ser associado a um dos pontos de inflexão do gráfico da isoterma de adsorção (Figura 3), a partir do qual passa a ocorrer um aumento significativo da quantidade de PHMB adsorvido nas membranas.

Comparando-se os dados das isotermas com os resultados mostrados na Figura 1, nota-se que, a baixas concentrações (de até cerca de $0,012 \mathrm{~mol} / \mathrm{L}$ ), a diminuição exponencial da eficiência de incorporação deve ocorrer devido à adsorção do PHMB em monocamada, exaurindo progressivamente os sítios disponíveis para adsorção na superfície da matriz. A maiores concentrações, as moléculas de PHMB passam a adsorver em multicamadas sobre as moléculas de PHMB já adsorvidas. A partir deste ponto, a concentração de PHMB torna-se alta em relação aos sítios disponíveis para adsorção, diminuindo a eficiência de incorporação, mas não a fazendo tender a zero por não se atingir na faixa estudada o platô estimado de máxima adsorção, estimado em 3,19 mol/kg (equivalente a 7,97 g/g). 


\section{9 a 22 de outubro de 2014 \\ Florianópolis/SC}

\section{CONCLUSÕES}

Neste trabalho, verificou-se que a isoterma de adsorção de PHMB em membranas porosas de QA tem formato do tipo L-S, típico da adsorção de alguns surfatantes em interfaces sólido-líquido. O modelo geral para adsorção de surfatantes proposto por Zhu e Gu (1989) ajustou-se bem aos dados nas faixas de concentrações testadas, permitindo supor que a adsorção ocorre em monocamada a baixas concentrações de PHMB e na forma de hemimicelas ou mesmo admicelas à medida em que a concentração aumenta. Verificou-se que as membranas de Q-A possuem alta capacidade de adsorção de PHMB, permitindo impregnar altas quantidades do antimicrobiano, de até cerca de $6 \mathrm{~g}$ por grama de matriz polissacarídica nas condições testadas (máximo previsto de $8 \mathrm{~g} / \mathrm{g}$ ), no entanto, a eficiência de incorporação para se atingir esta quantidade adsorvida é baixa.

\section{REFERÊNCIAS BIBLIOGRÁFICAS}

ARCH BIOCIDES. Polyhexamethylene biguanide (PHMB). Product stewardship summary. 2008. Disponível em <http://www.archchemicals.com/Fed/Corporate/Docs/ACC/ACC_ PHMB.pdf >. Acesso em Abril/2014.

BLACKBURN, R.S.; HARVEY, A.; KETTLE, L.L.; PAYNE, J.D.; RUSSELL, S.J. Sorption of Poly(hexamethylenebiguanide) on cellulose: mechanism of binding and molecular recognition. Langmuir, v. 22, p. 5636-5644, 2006.

BUENO, C.Z.; MORAES, A.M. Development of porous lamellar chitosan-alginate membranes: Effect of different surfactants on biomaterial properties. J. Appl. Polym. Sci., v. 122, p. 624-631, 2011.

DE PAUlA, G. F.; NETTO, G. I.; MATTOSO, L. H. C. Physical and chemical characterization of poly(hexamethylene biguanide) hydrochloride. Polymers, v. 3, p. 928-941, 2011.

GAO, Y.; CRANSTON, R. Recent advances in antimicrobial treatments of textiles. Text. Res. J., v. 78, n. 1, p. 60-72, 2008.

O’MALLEY, L. P.; COLLINS, A. N.; WHITE, G. F. Biodegradability of end-groups of the biocide polyhexamethylene biguanide (PHMB) assessed using model compounds. J. Ind. Microbiol.

Biotechnol., v. 33, p. 677-684, 2006.

ZHANG, R.; SOMASUNDARAN, P. Advances in adsorption of surfactants and their mixtures at solid/solution interfaces. Adv. Colloid Interface Sci., v.123-126, p. 213-229, 2006.

ZHU, B.Y.; GU, T. General isotherm equation for adsorption of surfactants at solid/liquid interfaces. Part 1. Theoretical. J. Chem. Soc., Faraday Trans. 1, v. 85, n. 11, p. 3813-3817, 1989.

ZHU, B. Y.; GU, T.; ZHAO, X. General isotherm equation for adsorption of surfactants at solid/liquid interfaces. Part 2. Applications. J. Chem. Soc., Faraday Trans. 1, v. 85, p. 3819-3824, 1989.

AGRADECIMENTOS: À FAPESP, à CAPES e ao CNPq pelo apoio financeiro. 